\title{
Use of a hollow wire to facilitate angioplasty of occluded vessels
}

\author{
L D R SMITH, D KATRITSIS, M M WEBB-PEPLOE \\ From the Department of Cardiology, St Thomas's Hospital, London
}

SUMMARY An USCI 0.038 inch $(0.97 \mathrm{~mm})$ floppy tipped hollow wire was used to facilitate the passage of angioplasty guide wires across severe stenoses or vessel occlusions before balloon angioplasty. The hollow wire was passed through a standard 7 or 8 French gauge Judkins? coronary catheter to establish whether the obstruction could be breached. This reduced the cost ${ }_{\dot{\omega}}^{+}$ of failure because angioplasty guiding systems were not committed to procedures that were $\mathcal{O}^{\circ}$ unlikely to be successful. The hollow wire provides stability for the passage of the guide wire응 and can be used to measure distal pressure and inject contrast. To date it has been used in a total of 15 cases of occluded vessels; it failed to cross the lesion in four cases. Successful angioplasty $\frac{}{O}$ followed in all patients in whom the lesion was crossed.

Although primary success rates in percutaneous transluminal coronary angioplasty, even for multiple lesions and multivessel disease, ${ }^{1}$ are increasing, inability to cross the lesion remains a common cause of failure. Failure to cross a lesion is greatest with near or complete occlusion of a vessel. Because steerable balloon guide wires have floppy flexible tips to prevent local trauma it is difficult to apply appropriate force to cross the lesion with them.

Usually support for the balloon guide wire is increased by a balloon catheter, which can be advanced over the guide wire up to the proximal end of the stenosis or occlusion. The balloon catheter provides a stiffer, more stable platform from which to advance the wire, enabling more force to be delivered to the wire. Often, however, the inflexibility of the balloon prevents the force being applied in the right direction, and this is a critical factor in success. Even when the occlusion is crossed with the wire it may still be difficult or impossible to advance even an ultra-low profile balloon through the very small pathway. Furthermore, the use of a balloon involves a guiding catheter, balloon catheter, and balloon guide wire and the high failure rate ${ }^{2}$ means that expensive equipment is wasted if the lesion cannot be crossed. We describe a simple and inexpensive alternative that

Requests for reprints to Dr L D R Smith, Department of Cardiology, St Thomas's Hospital, London SE1 7EH.

Accepted for publication 13 December 1988 does not require a guiding system or balloon catheter and we report our experience of its use.

\section{Patients and methods}

We used a USCI 0.038 inch $(0.97 \mathrm{~mm})$ straight hollow wire with a flexible tapered tip (USCI3 catalogue number 006200 (fig 1). This hollow wire? can be passed through a routine 7 or 8 French left? or right Judkins' catheter and a balloon guide wire can in turn be passed through the hollow wire. If 0 this guide wire crosses the lesion, angioplasty can? be attempted and the hollow wire and coronary catheter can be exchanged for a guiding system andi balloon catheter. For such an exchange either an exchange wire or a spliceable wire is needed. $\mathrm{We}_{\mathrm{O}}$ have most commonly used a Schneider Medintag $\star د$ Kaltenbach 0.012 inch $(0.30 \mathrm{~mm})$ exchange wires (Schneider Medintag AG, lot number 30 003) with a J tip. ${ }^{3}$ The hollow wire will take the 0.014 inch $(0.36 \mathrm{~mm})$ version of the Kaltenbach wire but then small swelling at the tip of this size Kaltenbach wires is too large to pass into the hollow wire, so it cannot ${ }^{\omega}$ be exchanged without removing the ensemble.

The hollow wire was packed with an internal stiffening wire core that was removed before use. A screw-on accessory can be attached to allow flushing of the lumen, to lubricate movement of theo guide wire. The Kaltenbach wire was threaded into the hollow wire until the tip was sheathed within $\overline{\mathrm{C}}$ the floppy end of the hollow wire. The Kaltenbach ^Now Schneider Shiley. 


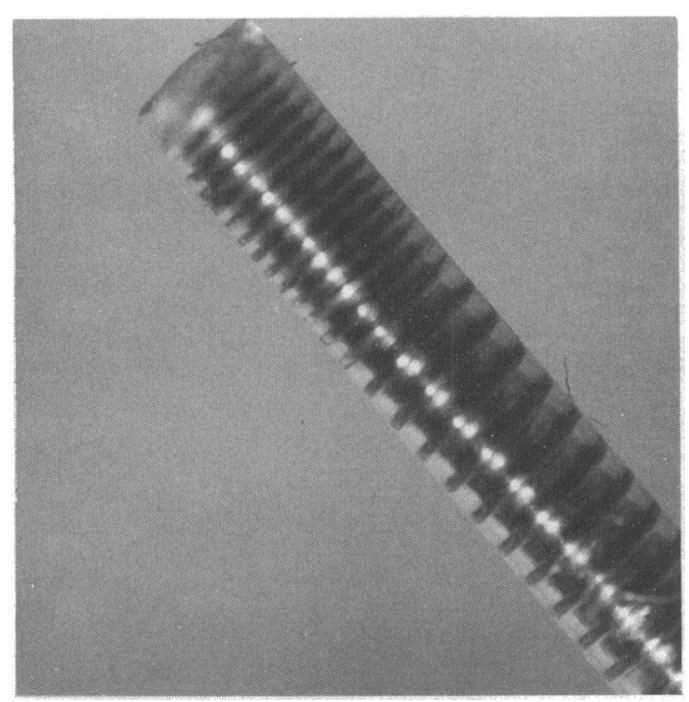

Fig 1 The tip of the USCI 0.038 inch $(0.97 \mathrm{~mm})$ hollow wire (catalogue number 006200) magnified 24 times. Guide wires of $0.012(0.30 \mathrm{~mm})$ and $0.014(0.36 \mathrm{~mm})$ inches easily pass through it.

wire-hollow wire ensemble was passed through a Tuohy-Borst haemostatic valve (ACS catalogue number 23242) and connected to the end of the diagnostic Judkins catheter that had been introduced into the femoral artery in the usual way via a short sheath. The side port of the haemostatic valve is attached to a manifold to allow pressure to be recorded or contrast injected through the Judkins catheter. The diagnostic catheter was engaged in the appropriate coronary artery ostium and the hollow wire and Kaltenbach wire were advanced to near the tip of the catheter. The Kaltenbach wire was then advanced along the appropriate artery to the occlusion with use of a torqueing tool if necessary. The hollow wire was then advanced very close to the tip of the Kaltenbach wire which could then be advanced further into the occlusion, with its tip protruding only one or two millimetres from the tip of the hollow wire. For every advance of the guide wire the hollow wire was further advanced, constantly providing support for the very delicate Kaltenbach tip. If the Kaltenbach wire successfully crossed the occlusion the hollow wire was usually able to follow it (fig 2). Then the guide wire could be removed and, after attachment of the screw-on flushing accessory, the hollow wire could be used to record distal pressure and to inject contrast into the distal vessel to show its appearance.

With the hollow wire still in situ across the lesion the Kaltenbach wire was replaced and the diagnostic Judkins catheter exchanged on the Kaltenbach wire-hollow wire ensemble with an appropriate guiding system. We found that this exchange was easier with the Kaltenbach wire-hollow wire ensemble than with the Kaltenbach wire alone since the increased stiffness prevents sudden displacement of the wire. This was especially true of the right coronary artery and graft angioplasty when it can be difficult to position securely the guiding catheter in the vessel orifice. When the guiding catheter was in place the hollow wire could be removed, leaving the Kaltenbach wire down the vessel. An appropriate balloon catheter was then advanced over the Kaltenbach wire. This invariably needs to be a balloon with an ultra-low profile because of the severity of the lesions underlying occlusion. Angioplasty then followed in the usual way.

\section{Results}

Between April and December 1987 we attempted percutaneous transluminal coronary angioplasty in 15 patients with chronic complete vessel occlusion. Two patients of the 15 had occlusions that were less than three weeks old and all but three occlusions had been present for more than four months. The mean time from presumed occlusion (on historical grounds) to angioplasty for all patients was five and a half months (range 3 weeks to 12 months) (failures, 10 months; successes, 4 months). Patients (13 men, two women) were aged between 41 and 70 years old (mean 53).

The sites of the lesions attempted were: nine in the left anterior descending coronary artery, one in the circumflex artery, three in the right coronary artery, and two in grafts. The table shows the results. In two patients ( 1 and 3 ) occlusion had not long been present

Table A summary of the results of angioplasty of vessel occlusions by the hollow wire

\begin{tabular}{ccclclc}
\hline Case & Sex & Age & $\begin{array}{l}\text { Vessel } \\
\text { occluded }\end{array}$ & $\begin{array}{l}\text { Duration } \\
(\text { mnth })\end{array}$ & Outcome & $\begin{array}{l}\text { Residual } \\
\text { stenosis } \\
\left({ }_{0}\right)\end{array}$ \\
\hline 1 & M & 46 & RCA & $<1$ & Success & 0 \\
2 & M & 46 & LAD graft & 7 & Success & 50 (large \\
graft)
\end{tabular}

RCA, right coronary artery; LAD, left anterior descending coronary artery; $\mathrm{Cx}$, circumflex. 


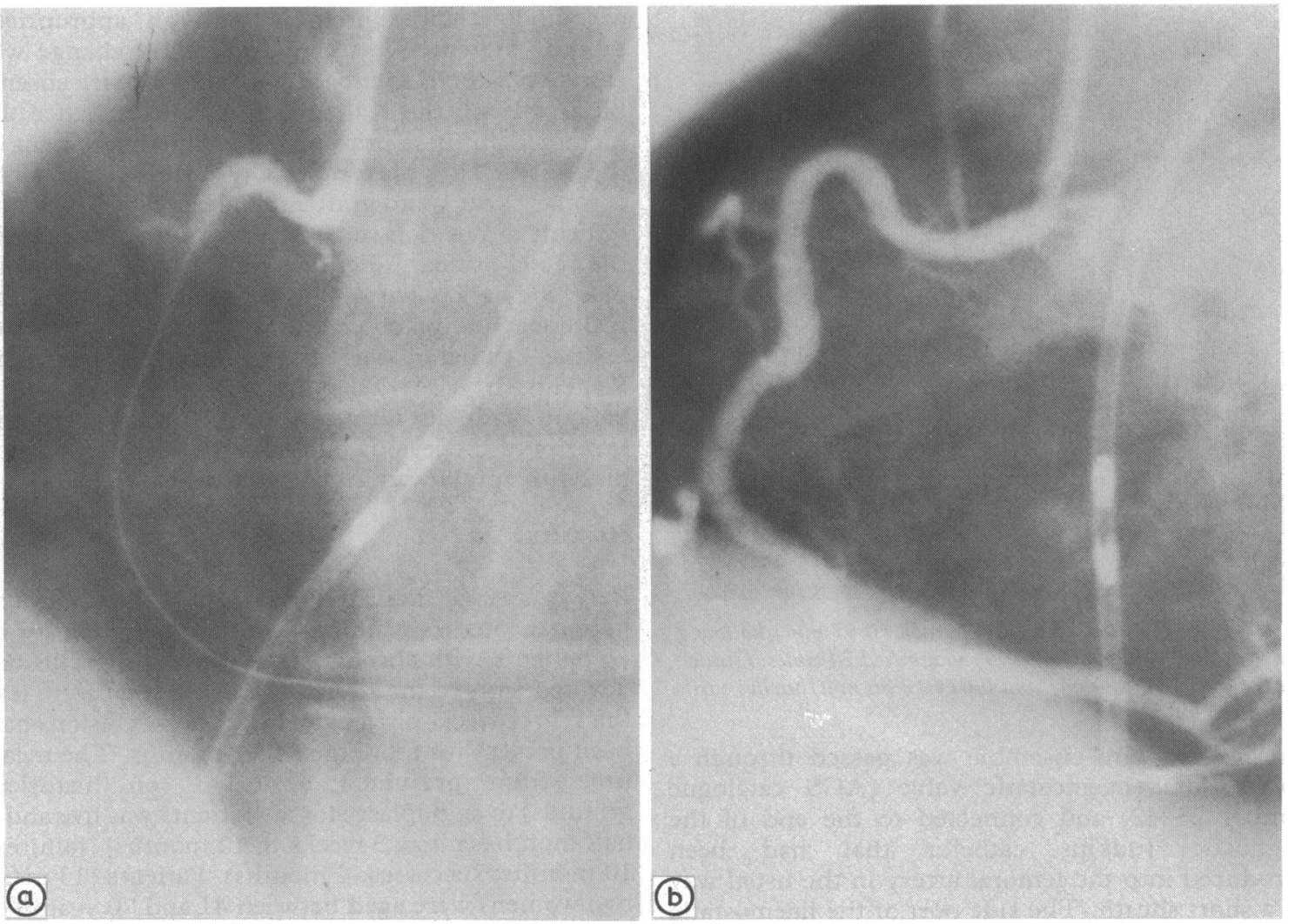

Fig 2 (a) The hollow wire passed through an occlusion of the proximal right coronary artery and on to the distal vessel. The tip of the Kaltenbach wire protrudes approximately one centimetre from the tip of the hollow wire. (b) The same right coronary artery after angioplasty of the proximal lesion.

and there was angiographic evidence of recent thrombus. In both these patients intracoronary thrombolysis (urokinase in patient 1 and streptokinase in patient 2) was used after initially breaching the occlusion. The results of successful breaching of the occlusion and angioplasty of the right coronary artery are shown in fig 2, while the left anterior descending artery is shown in fig 3.

\section{OTHER USES OF THE HOLLOW WIRE}

Contrast medium can be injected down the hollow wire to image the distal vessel. After removal of the Kaltenbach wire contrast can be injected through the hollow wire to delineate the distal artery.

We used the hollow wire to aid in the positioning of guard wires when we attempted lesions that affected side branches. Figure 3, showing two wires in position, is an example where the hollow wire was used to help to negotiate a severe stenosis of the left anterior descending artery that affected an important diagonal vessel. Initially a Kaltenbach guard wire was used and positioned in the diagonal branch. The left anterior descending coronary artery was then 8 dilated by a spliceable J wire and a $20 \mathrm{~mm}$ by $35 \mathrm{~mm}$ balloon.

costs

We originally used the hollow wire to save money. N When the lesion could not be crossed with the guideN wire, the attempt could be abandoned without wast- $N$ ing a guiding catheter and balloon catheter. The advantage of this approach can be seen from the prices listed below:

USCI 0.038 inch hollow wire

Schneider Medintag Kaltenbach exchange guide wire

Guiding catheter

Balloon catheter

Ultra-low profile balloon 

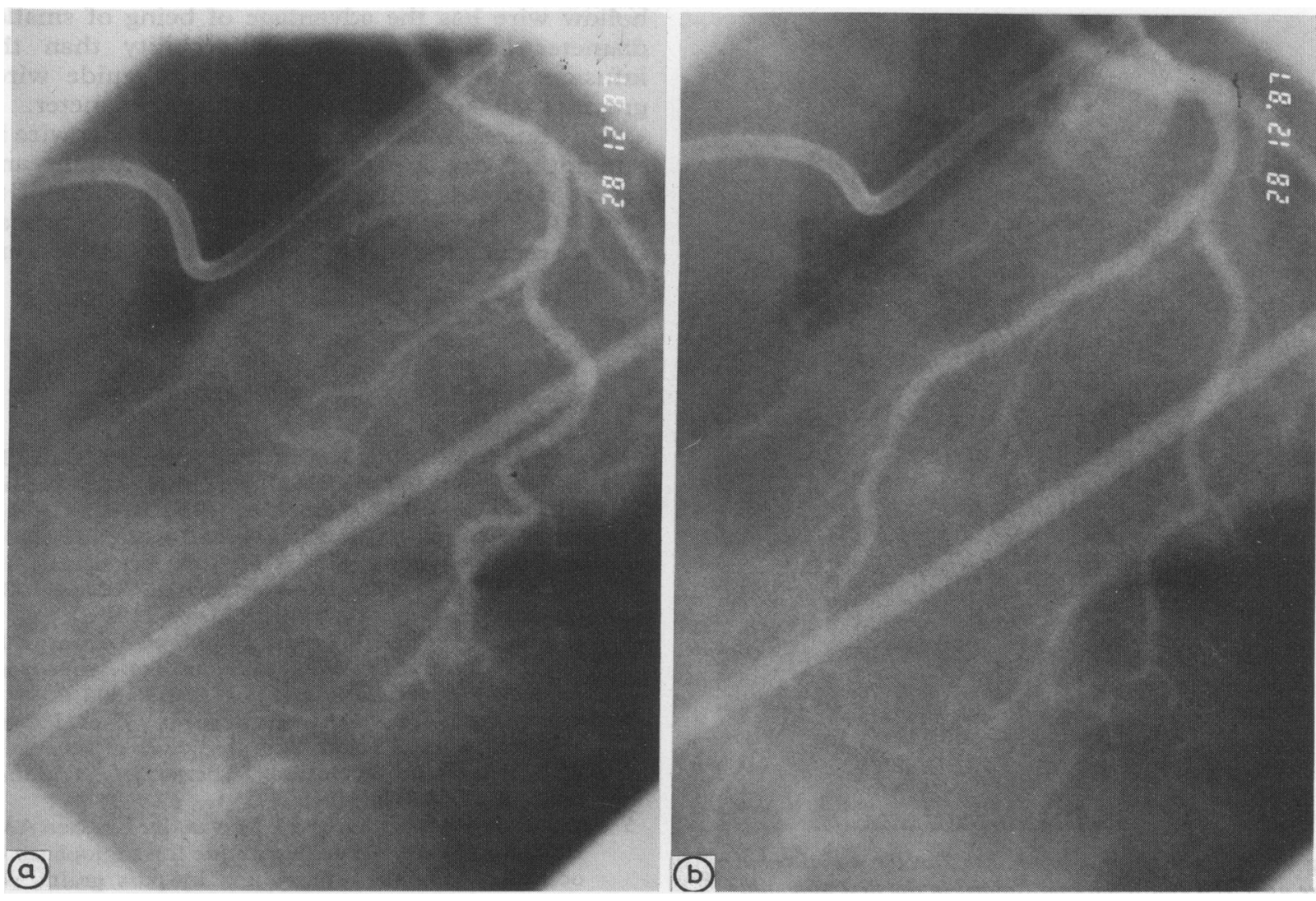

Fig 3 (a) A left craniocaudal view of left anterior descending coronary artery occluded just distal to the first septal branch. (b) The left anterior descending artery after disobliteration and angioplasty showing no residual stenosis and good distal flow.

Ordinary or high torque floppy guide wire

It costs $£ 160$ to use the hollow wire compared with $£ 465-£ 615$ for the guiding catheter and balloon catheter.

\section{Discussion}

Angioplasty of complete vessel occlusion is an attractive alternative to bypass surgery and is being increasingly attempted. Evidence suggests, as might be expected, that the sooner after occlusion that angioplasty is attempted the higher the chance of success. ${ }^{4}$ This is borne out in this small series in which the failures occurred with the oldest lesions and the most recent occlusions were easiest to reopen. We have shown a reasonable success rate with the older lesions, however. The success is to some degree attributable to the advantages of using the hollow wire. The hollow wire has several advantages: (a) increases the support and therefore the "pushs ability" of the guide wire; $(b)$ it is more manoeuvrable. and flexible than a balloon catheter; $(c)$ it can be used to record distal pressure and to inject contrast into the distal vessel; $(d)$ it crosses the iesion and because it has a larger diameter than the guide wire facilitates subsequent passage of a balloon cathete (e) it provides more support than an ordinary guide wire on which to exchange guiding catheters; and (f) it can be used initially through 7 or 8 French coronary catheters thus reducing costs.

A recent publication reports a similar techniqu这 with a $2 \cdot 5$ French gauge coronary infusion cathete instead of a hollow wire in nine patients. ${ }^{5}$ Like us, this group report on complete vessel occlusions not associated with acute myocardial infarction. Th8 


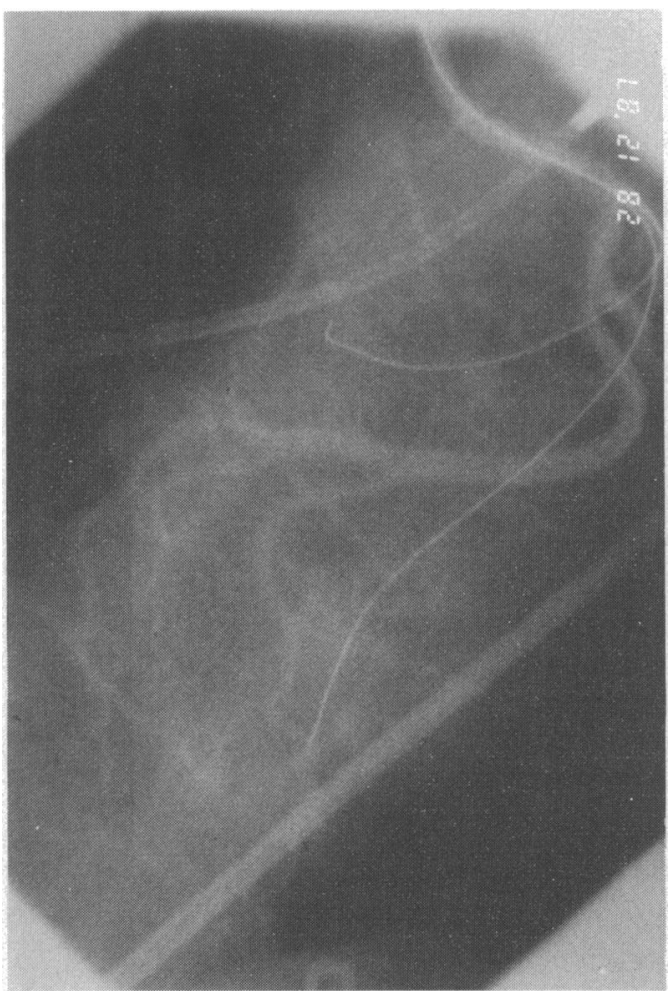

Fig $4 A$ left anterior oblique view showing a Kaltenbach wire used as a guard wire positioned in the first diagonal branch and a spliceable $J$ wire positioned in the left anterior descending artery. hollow wire has the advantage of being of smaller diameter and greater manoeuvrability than the infusion catheter but it cannot take guide wires greater than 0.014 inches $(0.36 \mathrm{~mm})$ in diameter.

We also recommend the use of the hollow wire in $\overline{0}$ attempting angioplasty of subtotal occlusions and $\bar{\sigma}$ severe stenoses that are expected to be difficult to $\frac{\bar{s}}{7}$ cross. The advent of detachable wire extensions may further reduce the cost of failure to cross the lesion.

\section{References}

1 Faxon DP, Kelsey S, Kellett MA, Ryan TJ, Detre K, and members of the NHLBI PTCA registry. Predic- $\omega$ tors of a successful angioplasty (NHLBI-PTCA reg- $\infty$ istry) [Abstract]. Circulation 1986;74(suppl II):193.

2 Clark DA. Complex PTCA II: total occlusions. In: Coronary angioplasty. New York: Alan R Liss, 1987: 63-8.

3 Kaltenbach $M$. The long wire technique-a new technique for steerable balloon catheter dilation of coronary artery stenosis. Eur Heart $J$ 1984;5:1004-9.

4 Wexman MP, Clark DA, Murphy MC, et al. Patient selection, complications and predictors of success in PTCA of total occlusion [Abstract]. Circulation 1986;72(suppl III):179.

5 de Swart JBRM, van Gelder LM, van der Krieken AM, El Gamal MIH. A new technique for angioplasty of $\varnothing$ occluded coronary arteries and by pass grafts, not $\overrightarrow{\vec{D}}$ associated with acute myocardial infarction. Cathet 3 Cardiovasc Diagn 1987;13:419-23. 